\title{
Characterization of Ni/Ti interlayer foil to assist diffusion bonding.
}

\author{
Emadinia, O. , Simões, S*, Viana, F. ${ }^{*}$ and Vieira, M.F.* \\ * CEMUC Department of Metallurgy and Materials Engineering, University of Porto, R. Dr. Roberto Frias, \\ 4200-465 Porto, PORTUGAL.
}

Email: emt10005@fe.up.pt

Joining of TiNi shape memory alloy is a challenging scientific and technological concern. To produce similar and dissimilar joints of TiNi it is necessary to develop further joining techniques that avoid or limit segregation, solidification cracking and distortion stresses, usually found in liquid phase welding processes. Diffusion bonding is a technique often selected for difficult to join materials. However, the high temperatures and pressures involved can cause other problems, like deformation, oxidation and phase transformation of the materials to join. The authors have explored the use of interlayers in diffusion bonding to achieve bonds at less demanding conditions with no alterations of the base materials [1]. Reactive multilayers deposited by sputtering can reduce the bonding conditions: temperature, pressure and time. The deposited nanostructured layers, with high levels of structural defects and large interfacial areas, enhance the diffusivity across the interface and improve bonding. In this investigation, interlayers of alternated $\mathrm{Ni} / \mathrm{Ti}$ foils, with equiatomic composition, were produced through accumulative roll bonding (ARB). One foil of Ni and Ti was stacked and rolled with a $50 \%$ reduction in thickness. Then, the produced bilayer foil was cut in two and subjected to another cycle. ARB interlayers were produced with 11 and 16 cycles with thickness ranging between 220 and $270 \mu \mathrm{m}$. Diffusion bonds of TiNi to Ti6Al4V, using the Ni/Ti interlayer foil, were produced at $800{ }^{\circ} \mathrm{C}$ during $60 \mathrm{~min}$, under a pressure of $10 \mathrm{MPa}$ in vacuum. The structure of the interlayer and diffusion bonds were characterized by scanning and transmission electron microscopy (SEM and TEM), electron backscattered diffraction (EBSD) and analysed by energy dispersive spectroscopy (EDS). SEM images of the multilayer foil cross section after 11 cycles of ARB did not reveal fragmented layers, as depict in Figure 1.a). Ultrafine and nanometric layers of $\mathrm{Ni}$ and $\mathrm{Ti}$ were observed after 16 cycles ARB Figure 1.b), as the result of the larger deformation. EDS analyses did not detect intermixing of $\mathrm{Ni}$ and $\mathrm{Ti}$ across their interface as displayed in the graph of Figure 1.c). These results were confirmed by EBSD analyses, see Figure 2.a), at $0.3 \mu \mathrm{m}$ from the interface only $\mathrm{Ni}$ is detected on the Ni layer; the quality of the Kikuchy patterns obtained on Ti layers was insufficient for indexation, making it impossible to analyze. The multilayer produced by ARB was also characterized by TEM, Figure 2b). TEM image of plane view samples show nanometric grains identified as $\mathrm{Ni}$, Ti and TiNi by electron diffraction. This results show that at a very small scale intermixing occurs and TiNi nanograins are formed. Diffusion bonding promotes the interdiffusion across the interface. At the centre of the interface, region of the $\mathrm{Ni} / \mathrm{Ti}$ interlayer, $\mathrm{SEM}$ images display areas with different atomic number contrast, Figure 3. Accordingly to EDS analyses the interface is constituted by $\mathrm{Ti}_{2} \mathrm{Ni}$, TiNi and $\mathrm{TiNi}_{3}$; a continuous layer of $\mathrm{Ti}_{2} \mathrm{Ni}$ is formed close to the $\mathrm{Ti} 6 \mathrm{Al} 14 \mathrm{~V}$ alloy.

This work was sponsored by FEDER funds through the program COMPETE - Programa Operacional Fatores de Competitividade - and by national funds through FCT under the project PEstC/EME/UI0285/2013.

[1] Simões, S. et al., Materials Science, 48, 7718-27, 2013. 


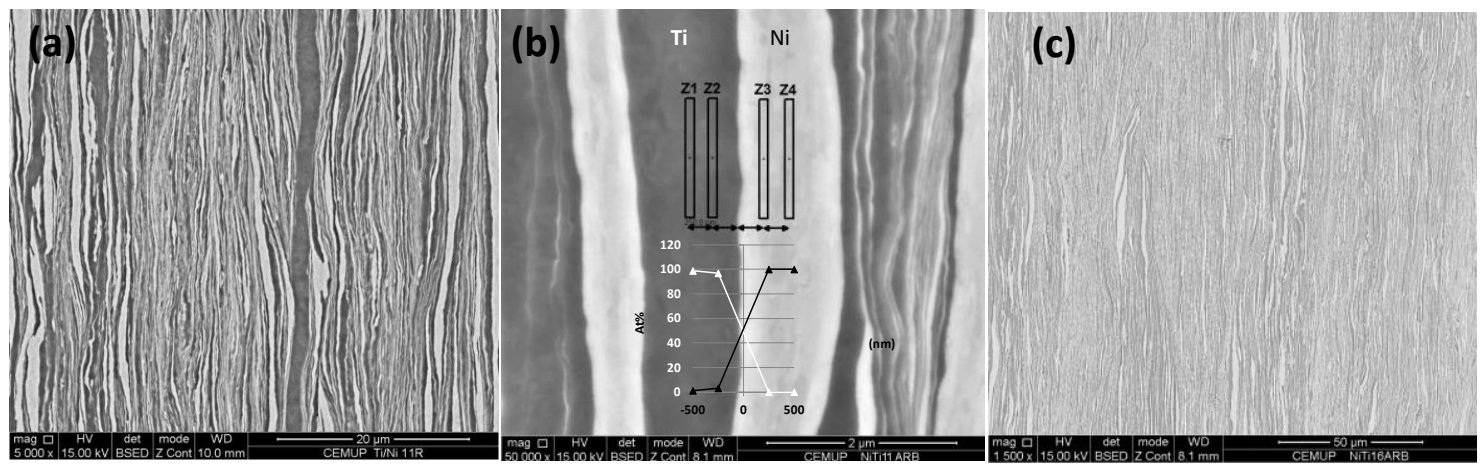

Figure 1. SEM images of the interlayers cross section (a) after 11, (b) after 16 ARB cycles, showing ultrafine and nanometric layers of $\mathrm{Ni}$ and $\mathrm{Ti}$; (c) variation of $\mathrm{Ni}$ and $\mathrm{Ti}$ composition across the interface (EDS analyses) after 11 ARB cycles.
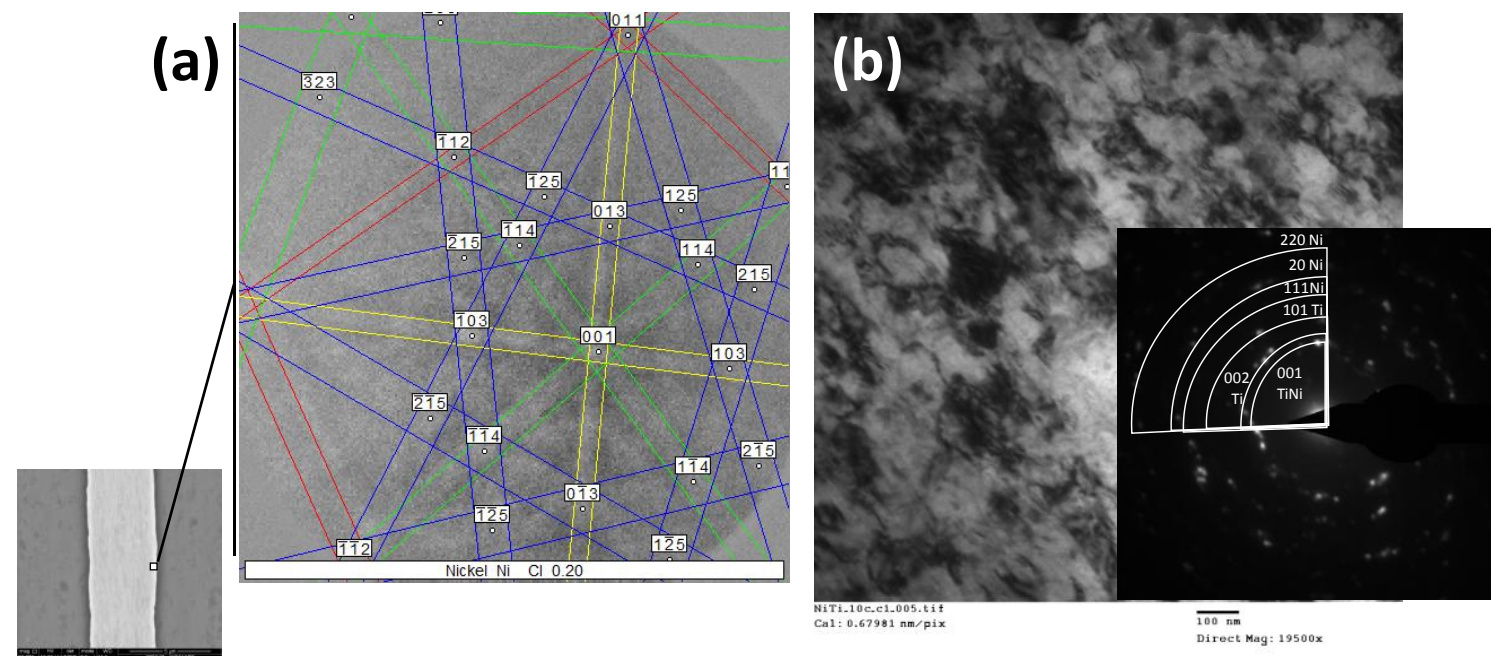

Figure 2. Ni/Ti interlayer after 11 ARB cycles: (a) Kikuchi pattern from the area shown in SEM image and (b) plane view TEM image showing nanometric grains and SAED spots indexed as Ni, Ti and TiNi.
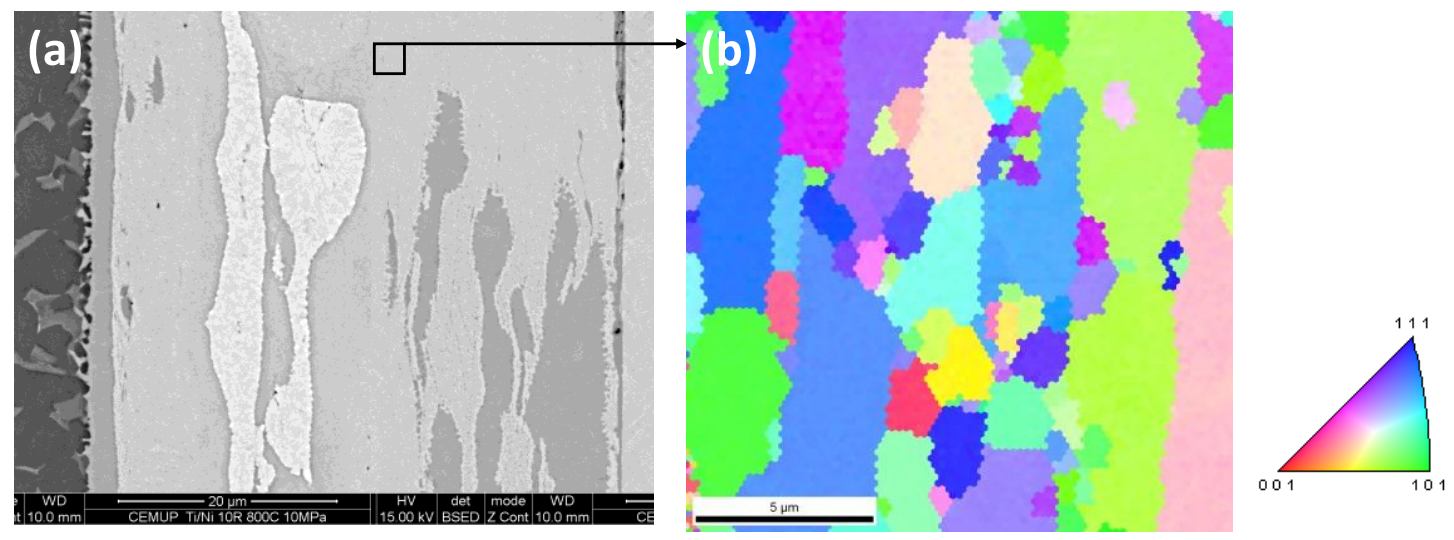

Figure 3. (a) SEM image of diffusion bonding interface produced with 11 ARB cycles interlayer and (b) EBSD orientation map showing grains of the identified zone. 